\title{
Effect of Total Sleep Deprivation on Visual Sequential Memory
}

\author{
Mohammad Habibur Rahman Khan', Salim Hossain ${ }^{2}$, Azmi Hassan ${ }^{3}$ \\ ${ }^{1}$ Institute for Community Health Development (iCODE), Universiti Sultan Zainal Abidin, Terengganu, Malaysia \\ ${ }^{2}$ Department of Psychology, University of Dhaka, Dhaka, Bangladesh \\ ${ }^{3}$ Faculty of Medicine, Universiti Sultan Zainal Abidin, Terengganu, Malaysia \\ Email:mhrkhan40@gmail.com
}

How to cite this paper: Khan, M. H. R., Hossain, S., \& Hassan, A. (2018). Effect of Total Sleep Deprivation on Visual Sequential Memory. Psychology, 9, 2337-2352. https://doi.org/10.4236/psych.2018.99133

Received: July 18, 2018

Accepted: September 10, 2018

Published: September 13, 2018

Copyright (C) 2018 by authors and Scientific Research Publishing Inc. This work is licensed under the Creative Commons Attribution International License (CC BY 4.0).

http://creativecommons.org/licenses/by/4.0/

\begin{abstract}
A substantial number of studies support the role of sleep deprivation (SD) on sleep-dependent memory processing. Our present study investigated the effect of Total Sleep Deprivation (TSD) on Visual Sequential Memory (VSM). Two-randomized group design was followed to collect the data where experimental group (33 adults) was kept totally sleep deprived for 24 hours a day and the control group (36 adults) was not. A subtest of Test of Memory and Learning (Reynolds \& Bigler, 1994) was used to measure VSM. Results revealed a significant decline in VSM for TSD $(t=3.914, p<0.001)$. Findings are discussed in the light of previous findings and theories in that VSM is the capability of an individual to perceive the visual properties of an object by eyes, a skill vitally necessary for many areas of work, like visual tracking, air traffic controlling, military task, marksmanship, screening, surgery, healthcare service, policing and so on.
\end{abstract}

\section{Keywords}

Sleep Deprivation, Total Sleep Deprivation, Visual Sequential Memory, Cultural Issue

\section{Introduction}

Visual sequential memory (VSM) is the ability of an individual to remember visual details related to sight of anything in correct sequence. Visual sequential memory or visual performance is the visual efficiency under ideal illumination. It is the ability of the eye to perceive or the visual properties of the object to be perceived. VSM describes a person's ability to remember a number of items, letters, numbers, or shapes in series. This skill is essential for many areas of life; 
such as hand-writing, cooking, remembering phone numbers, visual tracking, monitoring on-going display, sound sequencing and so on. It is, thus, the visual analysis skill within the larger framework of visual information processing (Borsting, 2006).

Sleep essentially contributes to memory. Sleep is essential to maintain brain's operative efficiency as shown by consequences of its deprivation, which may lead to accidents, cognitive errors, antisocial behaviours (Kamphuis, Meerlo, Koolhaas, \& Lancel, 2012; Cirelli \& Tononi, 2008; Alhola \& Polo-Kantola, 2007). However, researchers often differ regarding how to conceptualize the effects of sleep on memory. Evidences from a substantial number of studies support the role of sleep on sleep-dependent memory processing. Sleep affects procedural memory (Walker \& Stickgold, 2004). Patrick and Gilbert (1896) were the pioneers in reporting the performance impairing effects of total sleep deprivation (TSD). Since then, many researchers have extended these findings which indicate that TSD impairs many different cognitive functions including vigilance, working memory, short term memory, executive function, math processing, cognitive speed, spatial orientation, and motor control (Drummond, Anderson, Straus, Vogel, \& Perez, 2012; Rogers, Dorrian, \& Dinges, 2003; Smith, McEvoy, \& Gevins, 2002; Wright \& Badia, 1999).

Sleep deprivation has adverse effect to a variety of cognitive functions. Within working memory, some component processes are more vulnerable to SD than others. In a well-controlled study, Drummond et al. (2012) found that both one night of total sleep deprivation (TSD) and four nights of partial sleep deprivation (PSD) where subjects were allowed four hours of bed time at night, did not have any significant effect on visual working memory capacity. They observed TSD's negative impact only on performance in the filtering task where subjects had to ignore distracter stimuli in a visual scene (filtering efficiency) only. They suggest that components of visual working memory are differentially vulnerable to the effects of SD, and different types of SD influence visual working memory to the different degrees. Such findings have implications in operational settings where discriminating the relevant from irrelevant things is essential in taking decision such as look-out and watch-keeping duties. In visual memory, SD of one night did not have any effect on recognition (Alhola, Tallus, Kylmälä, Portin, \& Polo-Kantola, 2005). No effect was observed for visuospatial working memory after one night of sleep restriction (Nilsson et al., 2005).

Visual sequential memory (VSM) often termed as visuospatial sketchpad is one of the four components of working memory. The ability to selectively retain information in an accessible state called working memory. Baddeley (2000) suggested a model of working memory consists of four components; phonological loop, visuospatial sketchpad, episodic buffer and central executive. Sustained attention or vigilance is the executive processes of working memory (Baddeley, Cocchini, \& Della Sala, 1999). Attention and working memory are found to interact closely during encoding and manipulation (Fougnie, 2008). In measuring 
divided attention, performance was impaired after 24 hours of SD (Wright \& Badia, 1999). Detrimental effects on both speed and accuracy in measuring attention and working memory (WM) during SD were observed (Choo, Lee, Venkatraman, Sheu, \& Chee, 2005; Habeck et al., 2004; Jennings, Monk, \& Van der Molen, 2003). Impaired performance was observed in probed forced memory recall (Wright \& Badia, 1999), and memory search (McCarthy \& Waters, 1997).

Sleep deprivation (SD) impairs VSM. VSM is the quantitative assessment of the performance of a visual task, taking speed and accuracy into consideration. SD impairs visuomotor performance (Raidy \& Scharff, 2005), and the engagement of spatial attention (Bocca \& Denise, 2006). Decreased oculomotor functioning is also associated with impaired visual performance (De Gennaro, Ferrara, Curcio, \& Bertini, 2001), and sleepiness (Zils, Sprenger, Heide, Born, \& Gais, 2005). In well-controlled studies, it has been observed that the less sleep obtained due to sleep restriction, the more cognitive performance is impaired (Belenky et al., 2003; Van Dongen, Maislin, Mullington, \& Dinges, 2003). Research indicating that poor sleep impairs cognitive functions is particularly relevant to those with occupations with high visual task demands. These findings have significant implications for different operational settings where individuals may need to perform without adequate sleep. Jobs that involve receiving a range of visual information and discrimination of relevant cues from irrelevant things before taking action, such as visual tracking, baggage screening, air traffic controlling, military training, marksmanship, policing, surgery or healthcare service may also experience difficulties.

Sleep debt or sleep loss correlates with declined visual sequential memory, deteriorated alertness, declined psychomotor skills, slower problem-solving, and increased rate of false responding. Sleep deprivation weakens performance of visiomotor skills in measuring tasks of letter cancellation, digit symbol permutation, maze tracing or trail-making. Visual tasks is vulnerable to SD due to iconic memory possesses limited capacity and short duration (Raidy \& Scharff, 2005). SD impairs saccadic eye movements and the individual having sleep loss experiences difficulties in keeping rapid movement of eyes between points of fixation, thus, VSM is impeded for the impaired engagement of spatial attention (Bocca \& Denise, 2006), and oculomotor functioning (De Gennaro et al., 2001). Visual sequential memory loss is also manifested in psychomotor vigilance task (PVT), a reaction-timed task that requires sustained-attention in measuring the response-speed to a visual stimulus. VSM declining is also observed in both PVT decline and increased PVT lapses (Belenky et al., 2003). VSM is a critical intellectual capacity of an individual because keeping the images or clues in memory in proper sequence they were seen before is really critical to comprehension. Thus, loss of VSM impairs the ability to remember the proper sequence of words, letters, cues, or story narrative in correct order. These impairments in VSM are seen in our real life, such as, difficulty in remembering days of the week, months of the year, important telephone numbers, and in counting where 
the order of the elements is of higher importance. In reality, many students with reading difficulties have trouble with sequencing and poor sequential memory, which naturally affects their ability to read and spell in an accurate manner. When reading, individual with poor VSM may put letters in the wrong order, reading "reserve" as "reverse" or "felt" as "left", put words in wrong order, may omit letters like "cat" for "cart", may be unable to repeat longer words both orally or written.

\subsection{Rationale}

The importance of studying cognitive processes and cultural diversities is inevitable in order to understand human behaviour and cognition in a different cultural perspective. Sleep-cognition link is better understood where culture plays at large in understanding human psyche. Human psyche is better understood when laboratory studies on cognitive processes are supplemented with evidence from the particular culture in which a person lives (Wundt, 1916). People, in general, are quite unaware of cultural effect on perception. People often do not consider cultural effect on cognition in a great deal at all (Bonn, 2015).

There is a strong relationship between sleep and cognitive performances. The premise that sleep deprivation causes problems with behaviour, cognition, or other aspects of daytime functioning has long been discussed in western culture, US in particular. The perception towards sleep deprivation (SD) and its effects on visual sequential memory (VSM) in respect of cultural and communal diversities across the globe is assumed to vary considerably. Hence, study on this issue in other cultural context is inevitable in order to know more about SD-VSM link. Therefore, it is felt deemed necessary to investigate sleep deprivation and its effect on visual sequential memory in South Asian cultural context, particularly in Bangladeshi culture, from the point of developmental perspective.

In 2018, Bangladesh has become eligible for graduation from least developed country (LDC) to developing country (DC), and ascended her status to a new altitude. Bangladesh is the only country amongst the 47 LDCs that met all three criteria for graduation which include GNI per capita, Human Assets Index, and Economic Vulnerability Index (Khatun, 2018). The new status will certainly help in branding Bangladesh globally. Industrialization will be spread, new mill-factories will come into being, old factories will be renovated for the growth of production, privatization will be boomed and investors both local and foreign will be interested to invest in the country given her strength in certain areas such as the size of her exports, population, and above all Gross Domestic Product (GDP). Bangladesh has been transformed from an aid-dependent country into a trade-dependent one. This graduation brings forth a huge opportunities for Bangladesh attached with some challenges. The first challenge is, the overall economical capability has to be improved. This capability will be achieved through diversification of the economy, technological upgradation, human resource development, institutional strengthening and healthy working environ- 
ment. Second is, Bangladesh must improve its export competitiveness and diversify both markets and products for export. In order to access this, Bangladesh has to comply with strict conditions such as improved work conditions, health, women's empowerment, psycho-physical condition and sleep hygiene of workers.

Studies revealed that SD negatively affects cognitive performances, an ultimate attributes to human performance at work. Sleep is considered as a much contributing factor in figuring work effectiveness. Yet again, cross-cultural evidence on this issue is very scarce. Regrettably, no empirical data whatsoever have ever been found in Asian and African context, especially in the third world context, which justifies the need for further studies to see how SD influences cognitive functions and work performances amongst these peoples. Study on the sleep-cognition link in other developing context like Bangladesh would surely draw more concluding remarks in understanding the sleep dependent memory processing. Bangladesh's leading export goods are garments where workers' performances are based on visual sequential memory, visuomotor, and psychomotor skills. Thus, test of VSM would definitely be a milestone in this regard to determine work efficiencies related to sleep hygiene.

\subsection{Variables}

The variables taken in this study are as follows.

1) Independent variable: Total sleep deprivation of 24-hour a day including night-time sleep.

2) Dependent variable: Visual sequential memory.

\subsection{Objective of the Study}

The objective of the present study was to investigate whether there is any effect of total sleep deprivation (TSD) on visual sequential memory (VSM).

\subsection{Hypothesis}

It was hypothesized that there will be significant effect of total sleep deprivation on visual sequential memory. Statistically, it can be expressed as follows:

$$
\begin{aligned}
& \mathrm{H}_{0}: \mu_{1}=\mu_{2} \\
& \mathrm{H}_{1}: \mu_{1}>\mu_{2}
\end{aligned}
$$

\section{Methods}

\subsection{Participants}

Bangladeshi individuals both male and female were chosen as research population estimator for this study. A total of 100 individuals aged in between 18 and 40 years were selected as research participant. This age group is considered as the vital working force in any culture, and potential civil and military personnel are mostly young adults who belong to this age group (Miller \& Shattuck, 2005). Sleep requirements are fairly stable at around eight hours per night in 24-hour 
cycle of a day from the time individuals reach their mid-20s through their middle age years (Miller, Matsangas, \& Kenney, 2011), and is assumed to be common across the cultures. Therefore, the subjects chosen for this study are very much important since dynamic working force in civil and military in Bangladesh belong to this age group. Data required from respondents was 74. A total of 146 individuals were called out of which 100 volunteers were recruited based on some inclusion and exclusion criteria. Subjects were randomly assigned to experimental and control group, where experimental group was exposed to total sleep deprivation (TSD) for a day of 24 hours, and the control group was not, prior the test commencement. Subjects were given full one-night adaptation in the lab prior testing. After the adaptation night, test of VSM was administered on the subjects. After completion of the data collection procedure, data from 69 respondents were obtained in total on ground where 36 from control group and 33 from experimental group who completed full research procedure (Details given in "Procedure"). Distribution of samples is shown in Table 1.

\subsection{Psychometric Tools}

To collect the data following psychometrics tools were used:

1) Sleep tracker: A wrist-worn sleep tracker cum actimeter has been used to track sleep and activity of the subjects taken for experimental group. The recent popular brand of actimeter plus sleep tracker named Xiaomi Mi Band (model-MI3 M4) has been chosen for this purpose. This wrist-worn sleep/activity tracker was used to have record of subjects' sleeping time in 24 hours a day. Each subject of experimental group had to wear this wrist-worn device for several days before taking them into sleep lab. This kind of device has been extensively used in US military, particularly in US Navy in conducting sleep research where sleeping and snoozing time of the participants are recorded (Miller, Shattuck, \& Matsangas, 2010; Matsangas \& Miller, 2006; Archibald, 2005). This device works on motion-sensing technology. The device has two components namely accelerometer and passive sleep tracker. Accelerometer is a sensor which measures the rate of change of movements in $\mathrm{X}, \mathrm{Y}, \mathrm{Z}$ axis. These information are then translated into data like walking or sleeping, and gets synced with the mobile application. For the sleep tracking part, it uses the passive sleep tracking which means software is used to calculate the pattern of sleep based on the information provided by the user in mobile application. This method of detecting the sleep pattern is not $100 \%$ accurate. However, if the purpose is to know whether the subject has slept or not, the device is fairly valid and reliable (Miller et al., 2010) (picture given in

Table 1. Distribution of samples in experimental and control group.

\begin{tabular}{cccc}
\hline Group & Male & Female & Total \\
\hline Control $\left(\mathrm{X}_{1}\right)$ & 21 & 15 & 36 \\
Experimental $\left(\left(\mathrm{X}_{2}\right)\right.$ & 8 & 25 & 33 \\
& & Grand Total & 69 \\
\hline
\end{tabular}




\section{Appendix 1).}

2) Sleep log: This is a self-reported record of an individual's sleeping and waking times with related information, usually over a period of several days. The information contains, for example, time of going to bed and waking up, total bed time, total nap time, total sleep time including nap a day, quality of sleep (very poor, poor, moderate, good and very good), frequency of going toilet at night, and use of caffeine, alcohol, cigarette \& sleeping pill before sleep. Subjects were provided with sleep log one week before the test to know their sleeping hygiene and culture. This kind of sleep log is extensively used by US Army, Navy and Airforce to keep log of the troops' sleeping time and pattern.

3) Visual sequential memory (VSM): VSM, a subtest of Test of Memory and Learning (TOMAL), a comprehensive memory battery developed by Reynolds and Bigler (1994) standardized in 2007 (Reynolds \& Voress, 2007), was chosen as a psychometric tool for this particular study. TOMAL is an individually administered, 14-subtest instrument designed to assess broad-band verbal and nonverbal memory functions across the age-span, narrow-band memory skills like paired-associate learning and spatial memory, and learning via changes in recognition and recall across multiple trials. VSM requires recall of the sequence of a series of meaningless geometric designs. The ordered designs are presented, followed by presentation of a standard order of the stimuli, and the examinee shows the order in which they originally appeared. Materials needed are Record Form, Picture Book, and a stopwatch. Subjects are shown abstract designs presented horizontally and are then required to indicate the order in which they were presented on a separate easel page when given the same designs in a random order. Examinee is briefed well before taking the test, if required first two items in the booklet can be shown and discussed. Thereafter, examiner has to proceed with the remaining items. Designs presented on each page are displayed for 5 seconds for the examinee to see. Scoring of this subtest based on the number of designs recalled in the correct position. For each correct answer, "1" point is awarded across all items, " 0 " for incorrect answer. The test terminates if the subject scores " 0 " for two items consecutively. Reliability: Internal reliabilities for the TOMAL are generally high, ranging from a low of 0.56 to a high of 0.98 . Nine of the fourteen core subtests have an average reliability in the 0.90 s, while the remaining five are in the 80 's. Test-retest reliability coefficients for the Core and Supplementary Indexes ranged from 0.81 to 0.92 . The fourteen subtest reliabilities averaged 0.81 . Validity: Content validity was assessed by reference to initial development and tryouts, task analysis, and representativeness. The exploratory factor analysis found that all TOMAL subtests had positive correlations with every other TOMAL subtest. The results of factor analysis show strong evidence for a measure of general memory and thus for TOMAL's Composite Memory Index (CMI). When compared to the WISC-R and the K-ABC, the TOMAL correlated in the mid 0.50 's.

4) Personal information form: A personal information form duly filled up 
by each subject was collected. This form contains data about subject's basic information including the history of past and present physical and mental health, record of previous work experience including night-shift, tobacco or alcohol habit, and use of any medication (specimen given in Appendix 2).

\subsection{Procedure}

An advertisement was circulated nationwide through daily newspapers both in native and English language for recruiting the participants. A total of 146 individuals responded to the call and showed their interest to be volunteer. Among them we recruited 100 respondents based on inclusion and exclusion criteria. However, we excluded those respondents who were reported physically or mentally sick, having reported past history of serious physical or mental illness, being on any long-term medications, alcoholic or any substance user, having any kind of sleep disorder, experience of regular night-shift work, have habitual irregular sleeping habits (sleep less than 6.5 hours/night), extreme morning or evening chronotype, having symptoms associated with sleep disorders. The respondents whose sleep tracker along with record in sleep log indicated habitual good sleep (sleep > 6.5 hours/night, sleeping no later than 1:00 AM and waking no later than 9:00 AM) entered the study after giving informed consent. It was assured that the participants did not smoke, consume any medications, stimulants, caffeine or alcohol for at least 24 hours prior testing.

Subjects were randomly assigned to experimental group where they were exposed to total sleep deprivation (TSD) for a day of 24 hours, and the control group who enjoyed regular sleep (6.5 hour > sleep at night). Once the participants were selected they were given a sleep log and a sleep tracker. In sleep log the participants manually recorded their sleep patterns (waking time, sleeping time etc.). Whereas, the sleep tracker automatically recorded the sleep time and activity time by using modern GIS technology. The experimental group was kept 24-hour a day sleep deprived prior data collection time. On the other hand, the control group was allowed to regular sleep. Participants were taken to the laboratory 12 hours before the test of VSM was administered. Subjects were allowed to follow their normal everyday activities in day time, which in turn ensured the ecological validity of the study (Dickinson, Drummond, \& McElroy, 2017; Sánchez-Ortuño, Edinger, Means, \& Almirall, 2010). Subjects were given full one-night adaptation in the lab prior testing. Once entered into the laboratory subjects' informed consents were taken for the study to carry out. Subjects were informed regarding all the aspects of the study and were assured of confidentiality of their personal information except for the research purpose only. Both the groups were looked after and accompanied by the research staffs during adaptation night and testing time.

We targeted to collect a complete data from 74 participants (37 experimental and 37 control) as desired. However, only 33 participants from experimental group and 36 participants from control group completed the total data collection 
procedure. Other participants failed to complete the study due to their unwillingness, feeling ill, failure to comply with the study protocol, and some other immediate emergencies. After they entered into the laboratory prior to 12 hours of data collection, the experimental group was not allowed sleeping. They were kept awaken while they read books, paper, or magazines. Some browsed on the smartphone, did social networking, listened music, and shared views with research staffs. On the other hand, the control participants were allowed to have their regular sleep.

After the adaptation night in lab, we administered VSM test on the subjects which took around 25 - 30 minutes to complete. The VSM is an individually administered and culture-free test. We hired eight postgraduate students as test administrators and trained them to administer the test. After completion of the test participants were thanked and given a token of memento. Thus, they were allowed to leave the laboratory.

\section{Results}

Data were analyzed using SPSS v.20. Descriptive and inferential analyses were carried out. The findings of the present study are presented in Table 2.

The findings indicate that there is a significant decline of visual sequential memory (VSM) of experimental group who were sleep deprived for 24-hour a day. This decrease in VSM is significant $(p<0.001)$.

\section{Discussion}

It was hypothesized that sleep deprivation (SD) would greatly affect the test scores of experimental subjects. It was believed that the research subjects who were totally sleep deprived for a 24-hour day would score significantly less on the visual sequential memory (VSM) than those who obtained normal sleep the night prior. The result demonstrates that total sleep deprivation (TSD) can lead to significant decline in performance of VSM task. Though the task is procedurally simple and relies on prior-learning, yet extended time of awakening leads to significant decline in VSM. The pattern of results with worsening performance across the test, is a frequently-observed pattern in the studies of sleep and human performance in VSM (Naghavi \& Nyberg, 2005; Baddeley, 2000). Attention and working memory are interrelated, which combines phonological loop, visuospatial sketchpad (visual sequential memory), episodic buffer and central executive-a human working memory model suggested by Baddeley (2000). In VSM, sustained attention or vigilance is required which is otherwise the executive processes of working memory (Baddeley, Cocchini, \& Della Sala, 1999).

Table 2. Mean, standard deviation, and t-statistics of experimental and control group.

\begin{tabular}{ccccccc}
\hline Group & $N$ & $M$ & $S d$ & $d f$ & $t$ & Sig. \\
\hline Control $\left(\mathrm{X}_{1}\right)$ & 36 & 22.50 & 12.05 & & & \\
Experimental $\left(\mathrm{X}_{2}\right)$ & 33 & 13.27 & 6.44 & 67 & 3.914 & 0.000 \\
\hline
\end{tabular}


Attention and working memory interact closely during encoding and manipulation (Fougnie, 2008).

This empirical demonstration is merely a beginning of our investigation in a particular cultural context, Bangladesh, which would lead us to a computational cognitive process model in order to find a mechanistic explanation of the behaviour dynamics in future. The findings are strongly supported by ecological validity (Dickinson et al., 2017; Sánchez-Ortuño et al., 2010). Things are always assumed to be happened normally and can better be explained in natural settings rather than strict lab control where human subjects are observed. There are studies that have failed to find any effect under strict controlled condition (Drummond et al., 2012; Alhola et al., 2005; Nilsson et al., 2005).

\section{Conclusion}

The study claims that an important cognitive process in performing the VSM is the recalling of appropriate order of the designs or phenomena, and is more or less sleep-depended. Efficient visual perception is essential to recognize and remember letters, words, cues and their meaning. A deficiency related to this will lead an individual struggling with minor differences and a confusing state of brain function. Thus, recognizing, remembering and applying information quickly and easily become critical for performance. This hypothesis echoes the conclusions drawn from the studies that has explored that SD impedes visual performances (Bocca \& Denise, 2006; Raidy \& Scharff, 2005; Zils et al., 2005; Choo et al., 2005; Habeck et al., 2004; Walker \& Stickgold, 2004; Rinkenauer, Osman, Ulrich, Müller-Gethmann, \& Mattes, 2004).

The negative effect of sleep deprivation on attention and visual memory is supported by existing literatures. These results can be generalized to other people of same age group irrespective of cultural and geographical setting as VSM is largely reliant on a sleep-dependent memory consolidation process. Sleep-dependent mechanisms of neural plasticity lead to the consolidation of learning and memory. Stage-3 and stage-4 of NREM (non-rapid eye movement) sleep which is called slow-wave or deep sleep stage are primarily responsible for learning and memory consolidation. In TSD, a more thorough evaluation of higher cognitive functions is needed. Additionally, the effects of TSD have not been thoroughly compared among some essential subpopulations, for example, minor ethnical groups and females.

\section{Conflicts of Interest}

The authors declare no conflicts of interest regarding the publication of this paper.

\section{References}

Alhola, P., \& Polo-Kantola, P. (2007). Sleep Deprivation: Impact on Cognitive Performance. Neuropsychiatric Disease and Treatment, 3, 553-567. 
Alhola, P., Tallus, M., Kylmälä, M., Portin, R., \& Polo-Kantola, P. (2005). Sleep Deprivation, Cognitive Performance, and Hormone Therapy in Postmenopausal Women. $\mathrm{Me}$ nopause, 12, 149-155. https://doi.org/10.1097/00042192-200512020-00008

Archibald, K. (2005). Effects of Noise, Temperature, Humidity, Motion and Light on the Sleep Patterns of the Crew of the HSV-2 SWIFT. Monterey, CA: Naval Postgraduate School.

Baddeley, A. (2000). The Episodic Buffer: A New Component of Working Memory? Trends in Cognitive Sciences, 4, 417-423. https://doi.org/10.1016/S1364-6613(00)01538-2

Baddeley, A., Cocchini, G., \& Della Sala, S. (1999). Working Memory and Vigilance: Evidence from Normal Aging and Alzheimer's Disease. Brain and Cognition, 41, 87-108. https://doi.org/10.1006/brcg.1999.1097

Belenky, G., Wesensten, N. J., Thorne, D. R., Thomas, M. L., Sing, H. C., Redmond, D. P., Russo, M. B., \& Balkin, T. J. (2003). Patterns of Performance Degradation and Restoration during Sleep Restriction and Subsequent Recovery: A Sleep Dose-Response Study. Journal of Sleep Research, 12, 1-12. https://doi.org/10.1046/j.1365-2869.2003.00337.x

Bocca, M. L., \& Denise, P. (2006). Total Sleep Deprivation Effect on Disengagement of Spatial Attention as Assessed by Saccadic Eye Movements. Clinical Neurophysiology, 117, 894-899. https://doi.org/10.1016/j.clinph.2006.01.003

Bonn, B. G. (2015). Why Culture Matters: Social Context and How We Make Sense of Our Lives. In S. Haque, \& E. Sheppard (Eds.), Culture and Cognition: A Collection of Critical Essays (pp. 67-73). Switzerland: Peter Lang.

Borsting, E. (2006). Overview of Vision Development. In M. Scheiman, \& M. Rouse (Eds.), Optometric Management of Learning-Related Vision Problems (p. 43). St. Louis: Mosby.

Choo, W. C., Lee, W. W., Venkatraman, V., Sheu, F. S., \& Chee, M. W. (2005). Dissociation of Cortical Regions Modulated by Both Working Memory Load and Sleep Deprivation and by Sleep Deprivation Alone. Neuroimage, 25, 579-587. https://doi.org/10.1016/j.neuroimage.2004.11.029

Cirelli, C., \& Tononi, G. (2008). Is Sleep Essential? PLoS Biology, 6, e216. https://doi.org/10.1371/journal.pbio.0060216

De Gennaro, L., Ferrara, M., Curcio, G., \& Bertini, M. (2001). Visual Search Performance across $40 \mathrm{~h}$ of Continuous Wakefulness: Measures of Speed and Accuracy and Relation with Oculomotor Performance. Physiology \& Behaviour, 74, 197-204. https://doi.org/10.1016/S0031-9384(01)00551-0

Dickinson, D. L., Drummond, S. P. A., \& McElroy, T. (2017). The Viability of an Ecologically Valid Chronic Sleep Restriction and Circadian Timing Protocol: An Examination of Sample Attrition, Compliance, and Effectiveness at Impacting Sleepiness and Mood. PLoS One, 12, 1-17. https://doi.org/10.1371/journal.pone.0174367

Drummond S. P., Anderson, D. E., Straus, L. D., Vogel, E. K., \& Perez, V. B. (2012). The Effects of Two Types of Sleep Deprivation on Visual Working Memory Capacity and Filtering Efficiency. PLoS One, 7, e35653. https://doi.org/10.1371/journal.pone.0035653

Fougnie, D. (2008). The Relationship between Attention and Working Memory. In N. B. Johansen (Ed.), New Research on Short-Term Memory (pp. 1-45). Hauppage, NY: Nova Science Publishers.

Habeck, C., Rakitin, B. C., Moeller, J., Scarmeas, N., Zarahn, E., Brown, T., \& Stern, Y. (2004). An Event-Related fMRI Study of the Neurobehavioural Impact of Sleep Deprivation on Performance of a Delayed-Match-to-Sample Task. Brain Research: Cognitive 
Brain Research, 18, 306-321. https://doi.org/10.1016/j.cogbrainres.2003.10.019

Jennings, J. R., Monk, T. H., \& Van der Molen, M. W. (2003). Sleep Deprivation Influences Some But Not All Processes of Supervisory Attention. Psychological Science, 14, 473-479. https://doi.org/10.1111/1467-9280.02456

Kamphuis, J., Meerlo, P., Koolhaas, J. M., \& Lancel, M. (2012). Poor Sleep as a Potential Causal Factor in Aggression and Violence. Sleep Medicine, 13, 327-334. https://doi.org/10.1016/j.sleep.2011.12.006

Khatun, F. (2018). LDC Graduation: What It Means for Bangladesh (p. 8). The Daily Star (Bangladesh).

Matsangas, P., \& Miller, N. L. (2006). The Effects of Ship Motion on the Sleeping Patterns of Crewmembers Aboard a High Speed Naval Vessel. Sleep, 29, A126.

McCarthy, M. E., \& Waters, W. F. (1997). Decreased Attentional Responsivity during Sleep Deprivation: Orienting Response Latency, Amplitude, and Habituation. Sleep, 20, 115-123. https://doi.org/10.1093/sleep/20.2.115

Miller, N. L., \& Shattuck, L. G. (2005). Sleep Patterns of Young Men and Women Enrolled at the United States Military Academy: Results from Year One of a Four Year Longitudinal Study. Sleep, 28, 837-841. https://doi.org/10.1093/sleep/28.7.837

Miller, N. L., Matsangas, P., \& Kenney, A. (2011). The Role of Sleep in the Military: Implications for Training and Operational Effectiveness. Department of Operations Research, Naval Postgraduate School, 4-14. Monterey: USA.

Miller, N. L., Shattuck, L. G., \& Matsangas, P. (2010). Longitudinal Study of Sleep Patterns of United States Military Academy Cadets. Sleep, 33, 1623-1631. https://doi.org/10.1093/sleep/33.12.1623

Naghavi, H. R., \& Nyberg, L. (2005). Common Fronto-Parietal Activity in Attention, Memory, and Consciousness: Shared Demands on Integration? Consciousness and Cognition, 14, 390-425. https://doi.org/10.1016/j.concog.2004.10.003

Nilsson, J. P., Söderström, M., Karlsson, A. U., Lekander, M., Akerstedt, T., Lindroth, N. E., \& Axelsson, J. (2005). Less Effective Executive Functioning after One Night's Sleep Deprivation. Journal of Sleep Research, 14, 1-6. https://doi.org/10.1111/j.1365-2869.2005.00442.x

Patrick, G. T. W., \& Gilbert, J. A. (1896). Studies from the Psychological Laboratory of the University of Iowa. On the Effects of Sleep Loss. Psychological Review, 3, 468-483. https://doi.org/10.1037/h0075739

Raidy, D. J., \& Scharff, L. F. (2005). Effects of Sleep Deprivation on Auditory and Visual Memory Tasks. Perceptual and Motor Skills, 101, 451-467. https://doi.org/10.2466/pms.101.2.451-467

Reynolds, C. R., \& Bigler, E. D. (1994). Test of Memory and Learning. Austin, TX: PRO-ED.

Reynolds, C. R., \& Voress, J. K. (2007). Test of Memory and Learning: Second Edition. Austin, TX: PRO-ED.

Rinkenauer, G., Osman, A., Ulrich, R., Müller-Gethmann, H., \& Mattes, S. (2004). On the Locus of Speed-Accuracy Trade-Off in Reaction Time: Inferences from the Lateralized Readiness Potential. Journal of Experimental Psychology: General, 133, 261-282. https://doi.org/10.1037/0096-3445.133.2.261

Rogers, N. L., Dorrian, J., \& Dinges, D. F. (2003). Sleep, Waking and Neurobehavioural Performance. Frontiers in Bioscience, 8, s1056-s1067. https://doi.org/10.2741/1174

Sánchez-Ortuño, M. M., Edinger, J. D., Means, M. K., \& Almirall, D. (2010). Home Is Where Sleep Is: An Ecological Approach to Test the Validity of Actigraphy for the As- 
sessment of Insomnia. Journal of Clinical Sleep Medicine, 6, 21-29.

Smith, M. E., McEvoy, L. K., \& Gevins, A. (2002). The Impact of Moderate Sleep Loss on Neurophysiologic Signals during Working-Memory Task Performance. Sleep, 25, 784-794. https://doi.org/10.1093/sleep/25.7.56

Van Dongen, H. P., Maislin, G., Mullington, J. M., \& Dinges, D. F. (2003). The Cumulative Cost of Additional Wakefulness: Dose-Response Effects on Neurobehavioural Functions and Sleep Physiology from Chronic Sleep Restriction and Total Sleep Deprivation. Sleep, 26, 117-126. https://doi.org/10.1093/sleep/26.2.117

Walker, M. P., \& Stickgold, R. (2004). Sleep-Dependent Learning and Memory Consolidation. Neuron, 44, 121-133. https://doi.org/10.1016/j.neuron.2004.08.031

Wright Jr., K. P., \& Badia, P. (1999). Effects of Menstrual Cycle Phase and Oral Contraceptives on Alertness, Cognitive Performance, and Circadian Rhythms during Sleep Deprivation. Behavioural Brain Research, 103, 185-194. https://doi.org/10.1016/S0166-4328(99)00042-X

Wundt, W. (1916). Elements of Folk Psychology: Outlines of Psychological History of the Development of Mankind. London: Allen \& Unwin (German Original 1912).

Zils, E., Sprenger, A., Heide, W., Born, J., \& Gais, S. (2005). Differential Effects of Sleep Deprivation on Saccadic Eye Movements. Sleep, 28, 1109-1115.

https://doi.org/10.1093/sleep/28.9.1109 
Appendix 1. Xiaomi Mi Band (Model-MI3 M4)

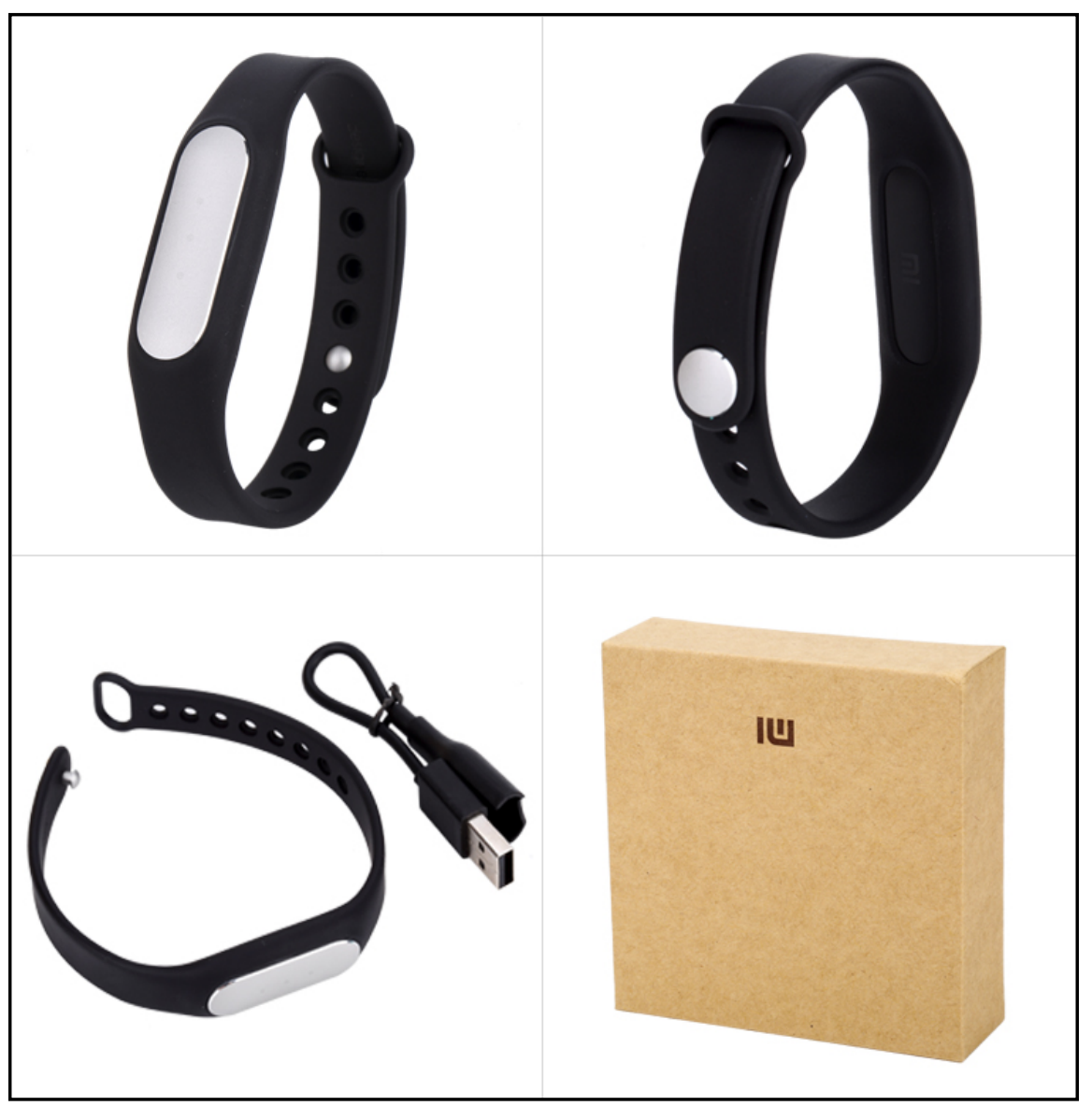




\section{Appendix 2. Personal Information Form}

COGNITIVE ABILITY TESTS FOR ADULTS

(English Version)

Code

Administrative Record Form for Adult

Name of the Person:

Height: .......... Weight: ..........kg Education:

Occupation:

Religion:

Marital Status:

Phone:

Sex: Male $\square \quad$ Female $\square$

Living: Village $\square \quad$ Small town $\square \quad$ Town $\square \quad$ District $\square \quad$ [tick $(\sqrt{ })$ ]

Name of the Test Administrator:

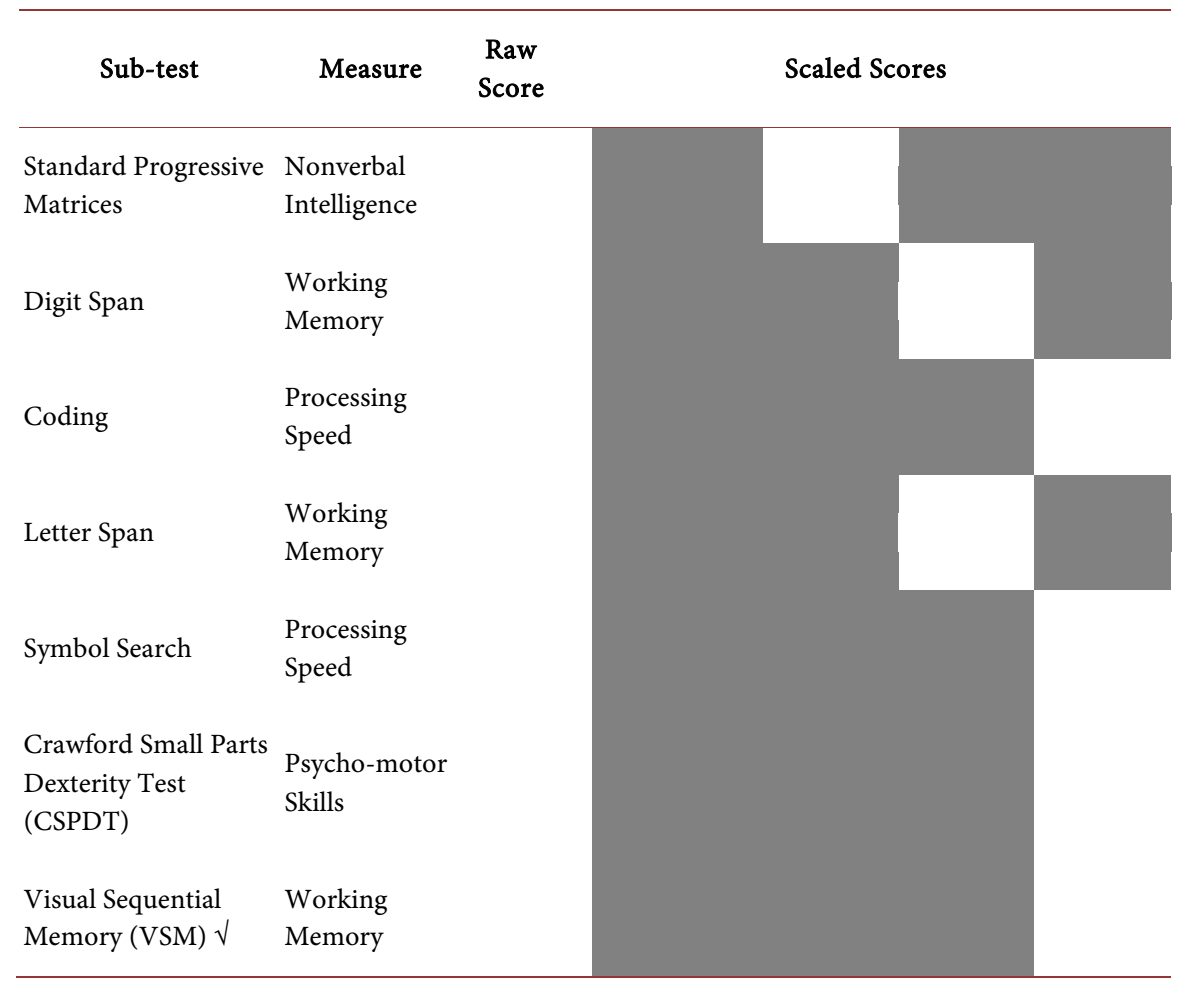




\section{(Put a tick $(\sqrt{ })$ and right in the applicable location)}

\begin{tabular}{|c|c|c|c|}
\hline No. & Ground & Yes & No \\
\hline 1. & Are you physically and mentally ready to participate in this research? & & \\
\hline 2. & $\begin{array}{l}\text { Have you slept last } 24 \text { hours? } \\
\text { If your answer is "yes", then mention the duration: .............................. hours/minute }\end{array}$ & & \\
\hline 3. & $\begin{array}{l}\text { Is it your habit to sleep less? } \\
\text { If your answer is "yes", then how many hours do you sleep daily? .................. hours }\end{array}$ & & \\
\hline 4. & Do you suffer from insomnia? & & \\
\hline 5. & $\begin{array}{l}\text { Are you suffering from any types of brain injury? } \\
\text { If yes, when }\end{array}$ & & \\
\hline 6. & $\begin{array}{l}\text { Are you suffering from Alzheimers, Dementia or any other mental disease? If so, then } \\
\text { mention }\end{array}$ & & \\
\hline 7. & $\begin{array}{l}\text { Are you suffering from any disease or physical illness right now? } \\
\text { If so, then mention those: }\end{array}$ & & \\
\hline 8. & $\begin{array}{l}\text { Are you taking any medicine for any reason? } \\
\text { If so, then mention those: }\end{array}$ & & \\
\hline 9. & $\begin{array}{l}\text { Did you ever get a hit at your brain in the past? } \\
\text { If so, then when? }\end{array}$ & & \\
\hline 10. & $\begin{array}{l}\text { Did you suffer from any deadly disease in the past? } \\
\text { If so, then when? }\end{array}$ & & \\
\hline 11. & Have you taken coffee, energy drink, alcohol or smoked in last 24 hours? & & \\
\hline 12. & Have you taken any chocolates/lozenge/toffee in last 24 hours? & & \\
\hline 13. & Have you recorded your daily activities in the Sleep Log that you were given? & & \\
\hline
\end{tabular}

